\title{
True Time Delay on Tunable Microwave Photonic Filter based on Stimulated Brillouin Scattering in Fibers
}

\author{
Juan Sancho ${ }^{(1)}$, Sanghoon Chin ${ }^{(2)}$, Mikel Sagues ${ }^{(3)}$, Alayn Loayssa ${ }^{(3)}$, Juan Lloret ${ }^{(1)}$, Ivana Gasulla ${ }^{(1)}$, \\ Salvador Sales ${ }^{(1)}$, Luc Thévenaz ${ }^{(2)}$ and José Capmany ${ }^{(1)}$ \\ (1) Optical and Quantum Communications Group, ITEAM Research Institute, Universidad Politécnica \\ de Valencia, Spain, email: juasandu@iteam.upv.es \\ ${ }^{(2)}$ Institute of Electrical Engineering, Ecole Polytechnique Fédérale de Lausanne, Switzerland, email: \\ luc.thevenaz@epfl.ch \\ ${ }^{(3)}$ Department of Electrical and Electronic Engineering, Universidad Pública de Navarra, Spain, email: \\ alayn.loayssa@unavarra.es
}

\begin{abstract}
A dynamically reconfigurable Microwave Photonic Filter based on True Time Delay generated by Stimulated Brillouin Scattering in optical fibers and separate phase shift of the optical carrier has been experimentally demonstrated, resulting in a flexible tunable Free Spectral Range.
\end{abstract}

\section{Introduction}

In next generation optical networks, tunable photonic delay lines will play an important role for broadband digital and analog communication systems. Recently, as a promising approach to provide all-optically controlled signal delaying, slow light (SL) techniques have been extensively investigated in various materials based on various physical mechanisms ${ }^{1,2}$. Slow light can be implemented as a robust solution for all-optical analogue signal processing ${ }^{3,4}$. In microwave photonics, tunable optical delay-lines are particularly required to implement microwave photonic filters (MPF) and to feed phased array antennas (PAA). These past few years, MPFs have been widely developed as a result of the considerable extension of their potential applications and also their inherent advantages compared to traditional RF circuits ${ }^{5}$.

In practice, only modest group delays can be achieved for broadband baseband signals, as already observed in digital signal delaying. However, in most typical microwave photonic systems, the RF subcarrier frequency is much higher than the bandwidth of the conveyed signal. Therefore, no information is carried in the broad frequency gap between the optical carrier and the single-sideband microwave envelope. Thus, the requirement of constant group (time) delay across the entire bandwidth can potentially be relaxed across this region as long as an appropriate phase-shift is also imparted to the carrier as already theoretically predicted in a previous study ${ }^{6}$, referred as the separate carrier tuning (SCT) technique.

In this context, we have experimentally demonstrated a reconfigurable MPF based on this SCT technique, using SBS-induced slow light and phase-shifting of the optical carrier by a fiber Bragg grating.

\section{SBS filter setup}

We implemented the basic configuration of a notch-type incoherent microwave photonic filter, which yields a periodic notch response. Fig. 1 depicts a simple structure of MPF, based on a Mach-Zehnder interferometer in which one arm contains a nominal delay. The transfer function of this two tap structure is given by ${ }^{5}$ :

$$
H(f)=a_{1}+a_{2} \cdot e^{-i(2 \pi f T)},
$$

where $a_{1}$ and $a_{2}$ are complex coefficients related to each filter tap and $T$ represents the filter's nominal delay. In principle, the notch depth depends on the amplitude difference between the two coefficients while the spectral periodicity is inversely proportional to the path delay imbalance on one arm with respect to the other, defining the free spectral range $F R S=1 / T$. Consequently, the tunability of the nominal delay $(T)$ is essentially required for a dynamic tuning of the FSR of such a transversal filter.

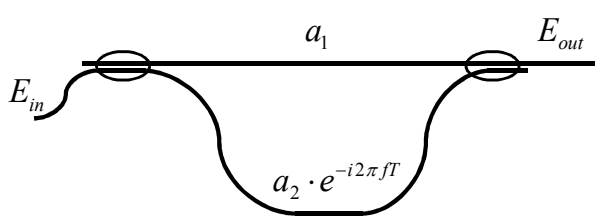

Fig. 1: Layout of the Microwave Photonic Filter, based on an unbalabced Mach-Zehnder interferometer.

The two-tap SBS tunable optical filter setup is depicted in Fig. 2. It consists of two main building blocks to reconfigure the frequency response of the MPF. The upper part of the diagram is dedicated to prepare and deliver the pump required for the implementation of the SBS-based tunable delay and the lower part realizes the tunable MPF itself.

The output from a $1550 \mathrm{~nm}$ distributedfeedback (DFB) laser is split using a 3dB fiberoptic coupler. The upper branch is electro- 


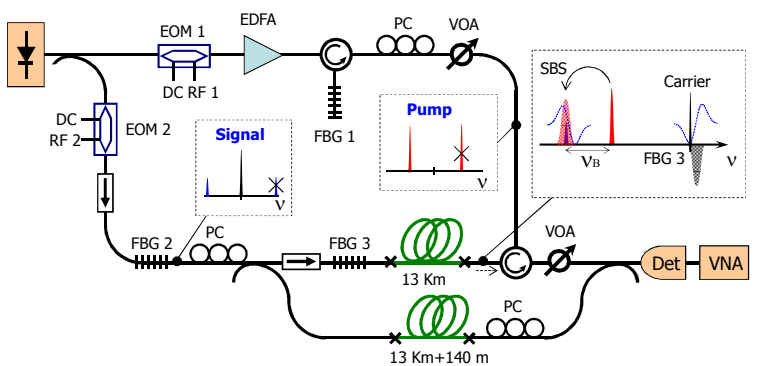

Fig. 2: Experimental setup

optically modulated (EOM1) over a $0-20 \mathrm{GHz}$ frequency range. The EOM1 bias point is properly adjusted to suppress in carrier. FBG1 is used to uniquely transmit the lower sideband, showing more than $20 \mathrm{~dB}$ suppression ratio between the two sidebands. The output signal is then amplified by an erbium doped fiber amplifier to be used as a controlled pump for the Brillouin gain generating the tunable delay. Its output power is controlled by a variable optical attenuator (VOA) for the all-optical control of the tunable delay generated by the SBS process.

In the lower branch, the light from the DFB laser is launched into EOM2, which is driven by the RF signal to be processed. FBG2 is used to remove the upper sideband, so as to result in a single sideband microwave signals. Then, the $\mathrm{RF}$ signal is launched into the $\mathrm{MPF}^{5}$ that operates under an incoherent regime. A circulator was used to allow the SBS pump signal to counter-propagate with respect to the OSSB-modulated carrier in one of the two branches of the MPF structure. The inset in Fig. 2 illustrates the spectral position of the Brillouin gain resonance generated by the pump and of the FBG3, which both induce a linear phase shift, in the spectral region of the microwave modulation and carrier, respectively. Consequently, the frequency of the RF signal modulating the EOM2 should be up-shifted by the Brillouin frequency shift $v_{B}$ with respect to the frequency of the RF signal modulating the EOM1. On the other hand, a proper optical phase tuning on the carrier is obtained using the phase spectral response of a third grating (FBG3). Finally, a Vector Network Analyzer (VNA) was employed to deliver a frequency swept RF input tone to the EOM2 and also to detect the output optical signal from the filter. This way the received RF signal is compared to the input to obtain the filter RF transfer function. A $140 \mathrm{~m}$-long optical fiber was inserted in one branch of the MPF to provide the target frequency response. In addition, the imbalance in optical path length between the two branches provides the condition of incoherent regime, but also, offers the possibility to study the feasibility of the SCT technique by investigating several resonances of the MWP filter. Polarization controllers $(P C)$ have been inserted to maximize the SBS response.

\section{Experimental results}

To demonstrate the tunability of the filter FSR, we first measured the effect of a pure phase shift on the optical carrier by recording the filter spectral transfer function. In this case it corresponds to a complex-valued two-coefficient $\mathrm{MPF}^{3-5}$.

Fig. 3 shows the expected pure shift of the

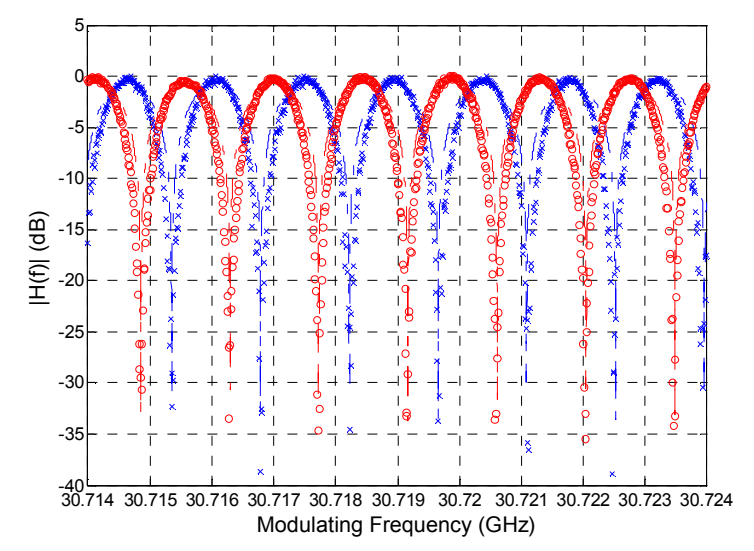

Fig. 3: Measured (circles and crosses) and simulated (dashed lines) filter frequency response tuning the FBG3 position.

spectral response of the MPF obtained with the SBS pump turned off and thus a fixed TTD, preserving the free spectral range and the spectral shape. This effect confirms that the phase shift imposed on the optical carrier propagating through the upper branch of the MPF can be changed by tuning the FBG3 central frequency. Two different phase shifts are shown with a difference of nearly 180 degrees. Experimental measurements show an excellent agreement with theoretical calculations. The two branches of the MPF have a path imbalance of $140 \mathrm{~m}$, corresponding to $700 \mathrm{~ns}$ and resulting ini a $1.43 \mathrm{MHz}$ FSR. Note that the central frequency of the filter notch is tuned while the shape of the filter transfer function remains unaltered, since only a fixed true time delay is introduced in one arm of the MPF.

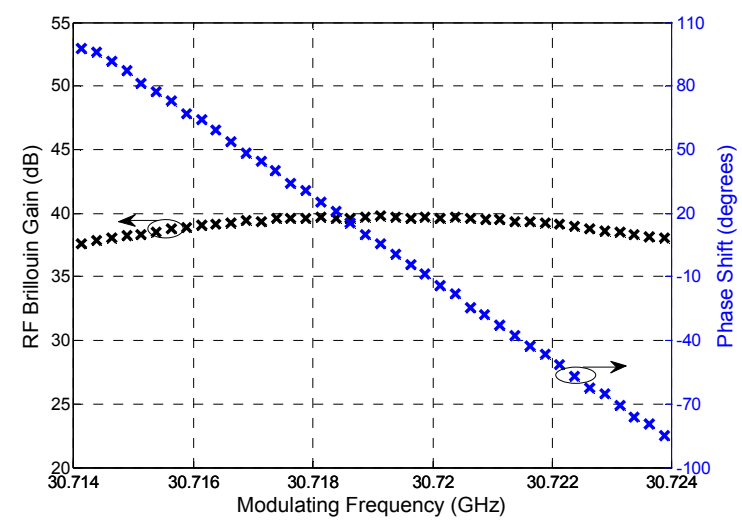

Fig. 4: SBS gain and phase measurements. 
For the Free Spectral Range tunability, we implemented a SBS-based tunable delay. A 13$\mathrm{km}$ dispersion shifted fiber (DSF) is used as Brillouin gain medium, showing a Brillouin frequency shift and a resonance bandwidth of $10.7 \mathrm{GHz}$ and $10 \mathrm{MHz}$, respectively. The microwave signal was generated at $30.7 \mathrm{GHz}$ through EOM2 and an RF tone of $20 \mathrm{GHz}$ was applied to EOM1 to match the Brillouin frequency of the fiber. Fig. 4 shows the spectral profile of the SBS gain resonance while the microwave subcarrier frequency is swept in the vicinity of the resonance, as well as the corresponding phase shift of the microwave signal with respect to the microwave signal frequency. A linear phase change was clearly observed with a moderate variation of the Brillouin gain. For a good performance of the MPF, it is critical to control not only the true time delay but also the amplitude coefficients of the filter, i.e. $a_{1}$ or $a_{2}$. To implement the TTD, a linear phase slope is required, since $T=1 /(2 \pi) d \square / d f$. In our case, the maximum achievable TTD value over the $10 \mathrm{MHz}$ span is 51.4 ns. However, the amount of induced true time delay can be yet tunable by simply varying the optical power of the Brillouin pump.

The SCT technique predicts that a TTD can be generated, which should result in a different

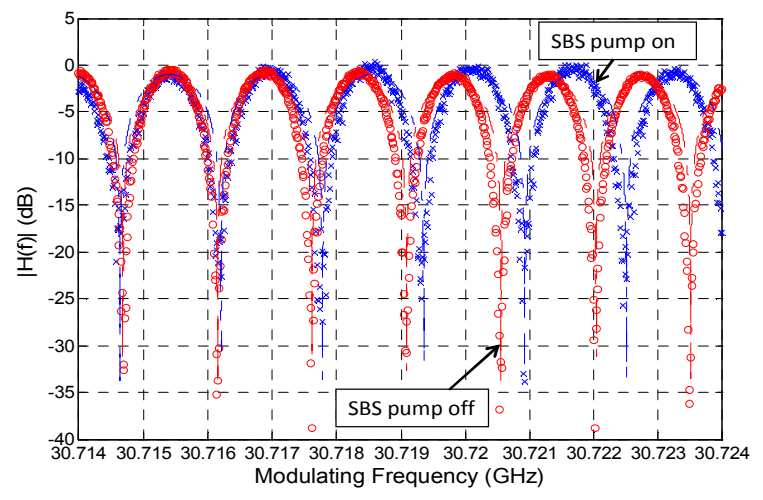

a)

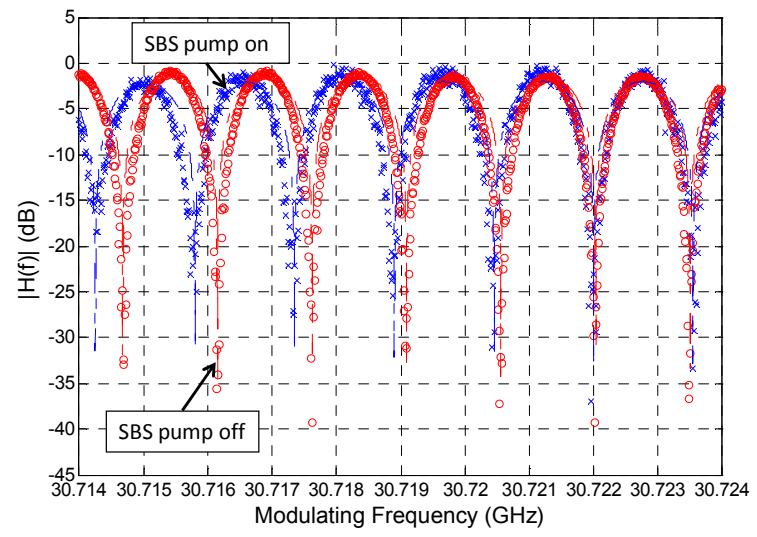

(b)

Fig. 5: Measured (circles and crosses) and simulated (dashed lines) FSR change on the filter frequency response for different FBG3 tuning positions (a) and (b). response of the MPF since its FSR will change upon tuning the delay. Fig. 5(a) and (b) confirms that the FSR of the filter changes when the pump is applied. When a $16 \mathrm{dBm}$ pump power is launched into the upper branch of the MPF, SBS generated a TTD of about $51.4 \mathrm{~ns}$. This means that the FSR will be increased up to $1.54 \mathrm{MHz}$. FBG3 is used for the carrier phase adjustment as required by the SCT technique ${ }^{8}$. The phase of the microwave signal can be described as:

$$
\phi\left(\omega_{R F}\right)=\phi\left(\omega_{c}\right)-\phi\left(\omega_{s}\right)+\left.\omega_{R F} \frac{\partial \phi\left(\omega_{c}\right)}{\partial \omega}\right|_{\omega_{c}-\omega_{s}}
$$

where $\phi\left(\omega_{\mathrm{c}}\right)$ and $\phi\left(\omega_{\mathrm{s}}\right)$ are the phase shifts at the carrier and sideband frequency respectively. To achieve TTD, $-60^{\circ}$ of carrier compensation is required in our particular case illustrated in Fig. 5(a). Tuning the central frequency of FBG3 also allows us to select the origin of the FSR variation. Fig. 5(b) shows an inverse FSR variation by inducing a $60^{\circ}$ carrier compensation. A notch filter with more than $30 \mathrm{~dB}$ extinction ratio has been achieved, showing an excellent agreement with theoretical prediction from Eq.(1). The authors wish to acknowledge the financial support of the FP7 project GOSPEL, the Microwave Photonics research Excellency award programme GVA PROMETEO 2008/092 and also the Plan Nacional I+D TEC2007-68065-C03-01.

\section{Conclusions}

A novel technique based on a reconfigurable MPF using the SCT technique and SBS slow light has been experimentally demonstrated. The effect of TTD induced by the SCT method was clearly verified through variation of the filter FSR. A simple two-tap filter has been used to demonstrate the technique, but the same approach can be easily extended to filters with an arbitrary number of coefficients. The main drawback is a limit in the FSR given by the spectral width of the Brillouin gain, which is of the order of a few tens of megahertz (typically 20-30 MHz at $1550 \mathrm{~nm}$ ). However, this system can be readily reconfigured to adapt broad signal bandwidths by modulating the pump ${ }^{7}$.

\section{References}

1 R. W. Boyd et al.,Prog.Optics 43, 497 (2002).

2 J. B. Khurgin et al. Slow light: Science and applications, CRC Press, Boca Raton, (2009).

3 M. Sagues et al., Opt. Exp. 16, 295 (2008).

4 W. Xue et al., IEEE Photon. Technol. Lett. 21, 167 (2009).

5 J.Capmany et al., Nature Photonics 1,319 (2007).

6 P. A. Morton et al, IEEE Photon. Technol. Lett. 21, 1686 (2009).

7 M. Gonzalez-Herraez et al., Opt. Exp. 14, 1395 (2006). 